\title{
Efficacy of acupuncture for dysphagia after stroke: a systematic review and meta-analysis
}

\author{
Yanyan $\mathrm{Lu}^{1,2}$, Ying $\mathrm{Chen}^{3}$, Dongting Huang ${ }^{2}, \mathrm{Ji} \mathrm{Li}^{4}$ \\ ${ }^{1}$ Guangzhou University of Chinese Medicine, Guangzhou, China; ${ }^{2}$ Department of Acupuncture and Massage, Guangxi Jiangbin Hospital, Nanning, \\ China; ${ }^{3}$ Department of Rehabilitation, Guangxi International Zhuang Medicine Hospital, Nanning, China; ${ }^{4}$ Department of Chinese Medicine, The \\ Second Affiliated Hospital of Guangxi Medical University, Nanning, China \\ Contributions: (I) Conception and design: Y Lu, Y Chen; (II) Administrative support: D Huang; (III) Provision of study materials or patients: J Li; (IV) \\ Collection and assembly of data: Y Lu, Y Chen, D Huang; (V) Data analysis and interpretation: Y Lu, Y Chen; (VI) Manuscript writing: All authors; \\ (VII) Final approval of manuscript: All authors. \\ Correspondence to: Ying Chen. Department of Rehabilitation, Guangxi International Zhuang Medicine Hospital, No. 8, Qiuyue Road, Wuxiang New \\ District, Liangqing District, Nanning 530200, China. Email: chenying3886@163.com; Dongting Huang. Department of Acupuncture and Massage, \\ Guangxi Jiangbin Hospital, 85 Hedi Road, Nanning 530021, China. Email: 1109010326@qq.com.
}

Background The risk of dysphagia after stroke is extremely high. The efficacy of acupuncture in the treatment of dysphagia after stroke lacks high-level evidence-based medical support. This study aimed to systematically evaluate the clinical value of acupuncture therapy in patients with dysphagia after stroke.

Methods: A electronic search of six databases were used to screen for randomized controlled trials (RCTs) of acupuncture treatment of patients with dysphagia after stroke. The search time was from the establishment of the database to 18 October 2020, and the search languages were limited to Chinese and English. The literature was screened and the data extracted independently by two researchers. The Cochrane System Evaluation Manual was used to evaluate the quality of the included literature.

Results: A total of 39 RCTs were included, of which 36 studies reported the effective rate of acupuncture treatment for dysphagia. The effective rate of the experimental group was higher than that of the control group [relative risk $(\mathrm{RR})=1.23,95 \%$ confidence interval $(\mathrm{CI}): 1.19$ to $1.27, \mathrm{P}<0.00001$ ]. The drinking test grading score of patients in the experimental group was lower than that of the control group [mean difference $(\mathrm{MD})=-0.75,95 \% \mathrm{CI}:-1.11$ to $-0.41, \mathrm{P}<0.0001]$ in 8 studies. The swallowing scores of patients in the experimental group were lower than those in the control group (MD =-4.63, 95\% CI: -5.68 to -3.59 , $\mathrm{P}<0.00001)$ in 8 studies. The Fujishima eating-swallowing rating score of the experimental group was higher than that of the control group [standardized mean difference (SMD) $=1.92,95 \%$ CI: 1.30 to 2.54 , $\mathrm{P}<0.00001]$ in 3 studies. The score of the dysphagia-specific quality of life scale of the experimental group was higher than that of the control group ( $\mathrm{SMD}=2.02,95 \% \mathrm{CI}: 0.82$ to $3.22, \mathrm{P}=0.0001)$ in 5 studies. The VFSS of the experimental group was higher than that of the control group (MD =2.53, 95\% CI: 1.89 to 3.17 , $\mathrm{P}<0.00001)$ in 5 studies.

Conclusions: The existing evidence supports that acupuncture therapy can significantly improve the swallowing function of patients with dysphagia.

Keywords: Stroke; acupuncture therapy; randomized control; meta-analysis; dysphagia

Submitted Dec 08, 2020. Accepted for publication Mar 23, 2021.

doi: 10.21037/apm-21-499

View this article at: http://dx.doi.org/10.21037/apm-21-499 


\section{Introduction}

Stroke encompasses both ischemic and hemorrhagic stroke and is the main cause of sudden death or disability worldwide. Ischemic stroke is often considered to be an infarction of the brain, spinal cord, or retina, accounting for about $71 \%$ of all strokes $(1,2)$. In some countries or regions, the incidence of ischemic stroke may be higher. An American survey (3) found that ischemic stroke accounts for $87 \%$ of all strokes in the United States. Both ischemic stroke and hemorrhagic stroke can cause local hypoxia in the brain tissue, followed by brain damage. For common ischemic strokes, effective thrombolytic therapy is usually required within 4.5 hours after the onset of disease (4). Beyond this time window, the brain may incur more serious damage. After onset of the disease, timely thrombolytic therapy can effectively reduce the complications caused by stroke (5). Common complications of stroke patients include hemiplegia, facial paralysis, speech disorder, and swallowing disorder (6-8). The risk of dysphagia after stroke is very high. Research (9) has reported that the incidence of dysphagia after stroke is about $37-78 \%$ which put patients at high risk of pneumonia, malnutrition and depression. Dysphagia severely affects the life and health of patients, causing pneumonia, dehydration, disability, or even death (10-14). At present, the treatment of swallowing dysfunction after stroke can have a certain effect. The treatment measures mainly include medication, traditional Chinese medicine, acupoint injection, biofeedback, and physical therapy (15-19). Acupuncture therapy in traditional Chinese medicine has been widely used in many diseases, and the efficacy of acupuncture in treating dysphagia after stroke has been reported in some studies (9-16). However, due to the possible differences between the diagnostic indicators and the methods of evaluating the efficacy of clinical researchers, the conclusions reached have all been based on small sample sizes and high-level evidence is lacking. In recent years, many novel studies on this topic have been published $(10,16)$. Therefore, this study aimed to systematically evaluate the efficacy of acupuncture in the treatment of dysphagia after stroke by collecting relevant literature, especially latest studies, so as to provide a theoretical basis for clinicians. We present the following article in accordance with the PRISMA reporting checklist (available at http://dx.doi.org/10.21037/apm-21-499).

\section{Methods}

\section{Inclusion and exclusion criteria}

The studies included in this meta-analysis were all randomized controlled trials (RCTs). The research participants included in the literature were those who were experiencing swallowing dysfunction after stroke. The intervention measures were mainly acupuncture and swallowing rehabilitation therapy, and the Kubota drinking water test, standardized swallowing assessment (SSA) score, Fujishima feeding-swallowing function grade score, swallowing disorder-specific quality of life scale (SWALQOL) score, and video fluoroscopic swallowing study (VFSS) were used to evaluate the clinical efficacy. Literatures were excluded if one of the following criteria were met: (I) unclear research object, and impossible to determine whether it was a patient with dysphagia after stroke treatment; (II) did not contain the outcome indicators of the study; (III) data could not be extracted or the full text was not found; (IV) repeated publication; $(\mathrm{V})$ review, abstract.

\section{Search strategy}

The search was conducted using keywords such as "stroke", "cerebral infarction", "cerebral hemorrhage", "acupuncture", "dysphagia", "random", "randomized".

\section{Literature screening and data extraction}

First, two researchers independently screened the retrieved documents according to the inclusion and exclusion criteria, and then any controversial documents were cross-checked by a third reviewer after evaluation and discussion to reach consensus. The two researchers individually extracted relevant information of the included literature, such as author's area, age, study type, sample size, intervention measures, outcome indicators, and so on.

\section{Literature quality evaluation}

As all the included studies were RCTs, the Cochrane System Review Manual was used for quality assessment, including: (I) randomization method; (II) group scheme hiding; (III) blind method; (IV) outcome evaluator and statistics; (V) 


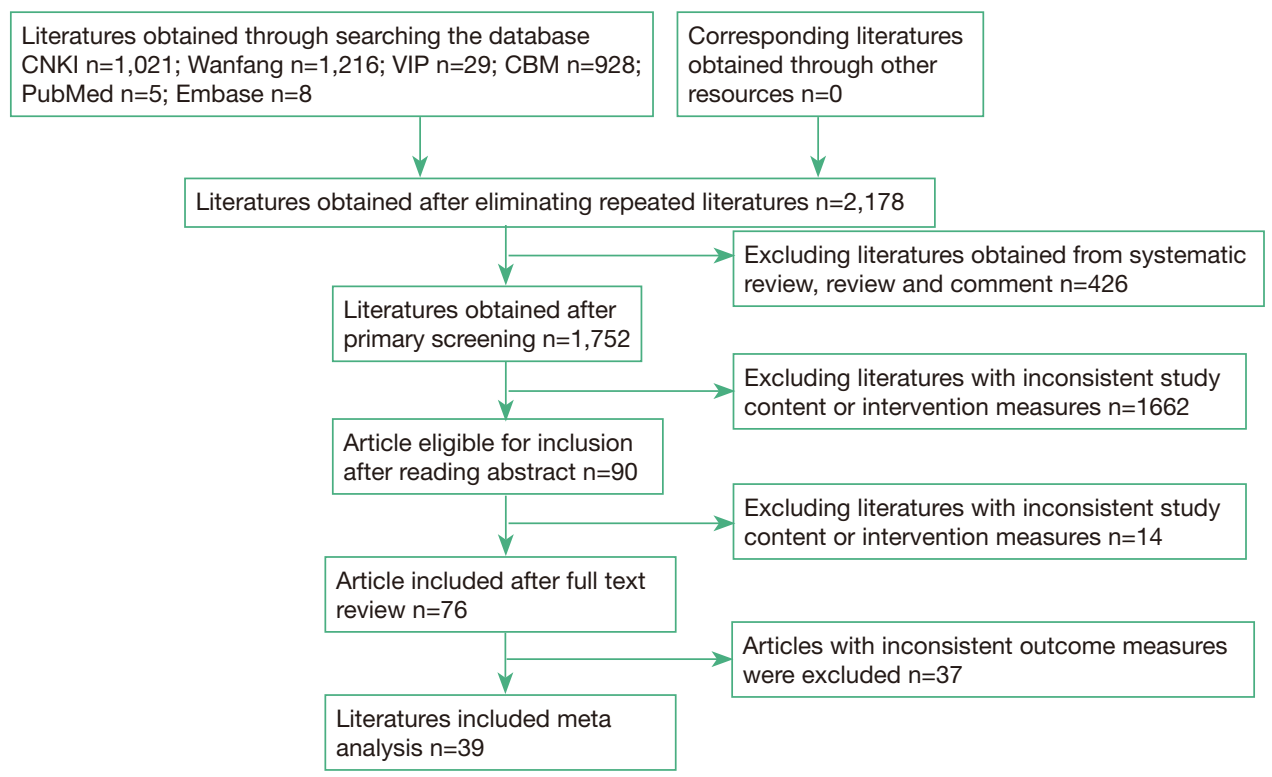

Figure 1 Document screening process and results. n, number; CNKI, China National Knowledge Infrastructure; VIP, weipu; CBM, Chinese BioMedical Literature Database;

risk of incomplete data processing; (VI) risk of selective reporting; (VII) other bias. The included literature was judged at the levels of "low risk", "high risk", and "unclear" from the above seven aspects, and drawn into a risk bias distribution map.

\section{Statistical methods}

RevMan 5.3 software (The Cochrane Collaboration, Copenhagen, Denmark) was used to conduct meta-analysis on the data, and the heterogeneity between the studies was determined by $\chi^{2}$ test combined with $\mathrm{I}^{2}$ quantitative analysis. If $\mathrm{P}>0.1$ and $\mathrm{I}^{2}<50 \%$, it was considered that there was no obvious heterogeneity among the included studies, and the fixed effects model was used for meta-analysis; if $\mathrm{P}<0.1$ and $\mathrm{I}^{2}>50 \%$, it was considered that there was obvious heterogeneity among the included studies. The publication bias of the included studies was judged by visual inspection of the symmetry of the funnel chart, and the symmetry of the scatter distribution indicated no obvious publication bias.

\section{Results}

\section{Literature}

According to the results of the literature search, 3,207 documents were obtained through preliminary examination. After a step-by-step screening, 39 documents were finally included, all of which were RCTs involving 3,078 patients. The literature screening process is shown in Figure 1.

\section{Basic characteristics and quality evaluation of literature}

The basic characteristics of the included studies are shown in Table 1. The quality evaluation of the included studies shows that some of the research quality indicators were specified in detail, but there were also cases in the literature that did not explain or clearly explain which made the literature have a high risk of bias. The percentage and distribution diagrams of the risk of bias in the study are shown in Figures 2 and 3.

\section{Meta-analysis}

\section{Treatment efficacy}

A total of 36 studies reported the effective rate of acupuncture therapy in the treatment of dysphagia, 1,433 cases in the experimental group and 1,413 cases in the control group. The 36 included studies were tested for heterogeneity, $\mathrm{I}^{2}=0 \%$ and $\mathrm{P}=0.80$, indicating that there was no heterogeneity among the included studies. Therefore, a fixed-effect model was used for meta-analysis. The results showed that the experimental group had a higher 
Table 1 Characteristic of included studies

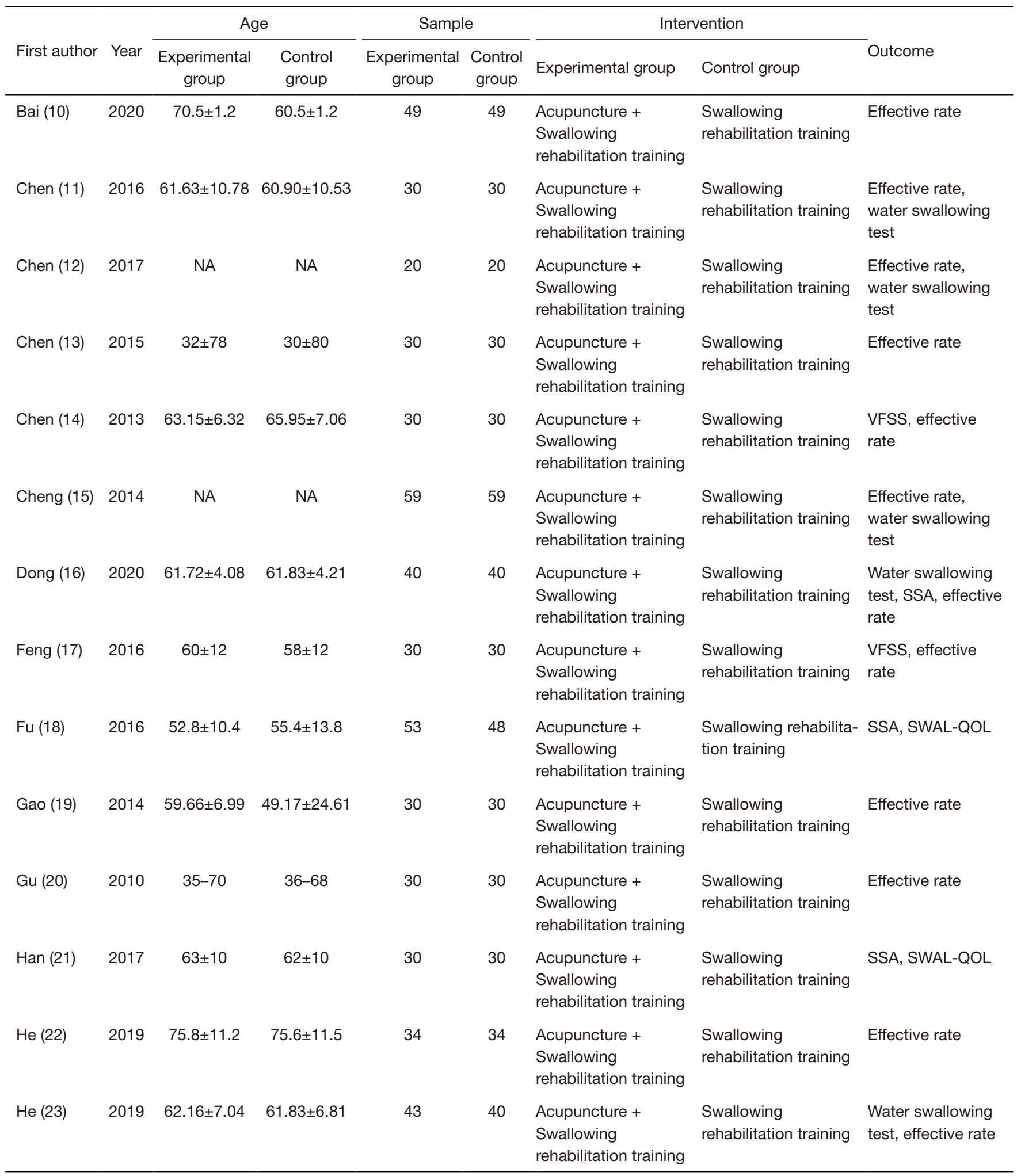

Table 1 (continued) 
Table 1 (continued)

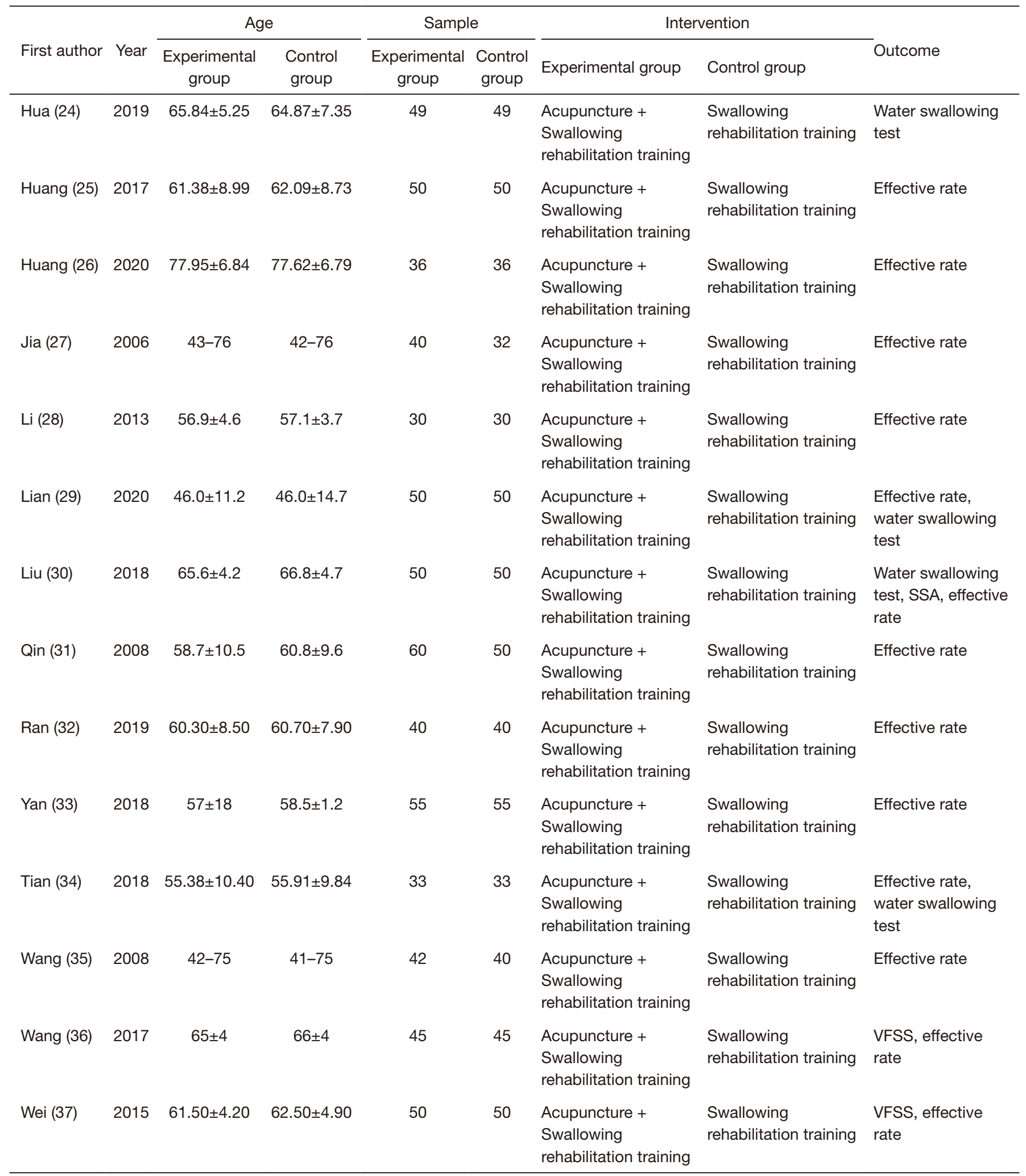

Table 1 (continued) 
Table 1 (continued)

\begin{tabular}{|c|c|c|c|c|c|c|c|c|}
\hline First author & Year & \multicolumn{2}{|c|}{ Age } & \multicolumn{2}{|c|}{ Sample } & \multicolumn{2}{|c|}{ Intervention } & Outcome \\
\hline Yu (40) & 2012 & $63 \pm 10$ & $64 \pm 11$ & 40 & 38 & $\begin{array}{l}\text { Acupuncture + } \\
\text { Swallowing } \\
\text { rehabilitation training }\end{array}$ & $\begin{array}{l}\text { Swallowing } \\
\text { rehabilitation training }\end{array}$ & Effective rate \\
\hline Zhang (42) & 2011 & NA & NA & 30 & 30 & $\begin{array}{l}\text { Acupuncture + } \\
\text { Swallowing } \\
\text { rehabilitation training }\end{array}$ & $\begin{array}{l}\text { Swallowing } \\
\text { rehabilitation training }\end{array}$ & Effective rate \\
\hline Zhang (43) & 2018 & $61 \pm 11$ & $59 \pm 9$ & 30 & 30 & $\begin{array}{l}\text { Acupuncture + } \\
\text { Swallowing } \\
\text { rehabilitation training }\end{array}$ & $\begin{array}{l}\text { Swallowing } \\
\text { rehabilitation training }\end{array}$ & Effective rate \\
\hline Zhen (44) & 2017 & $61.40 \pm 4.20$ & $60.80 \pm 4.60$ & 44 & 43 & $\begin{array}{l}\text { Acupuncture + } \\
\text { Swallowing } \\
\text { rehabilitation training }\end{array}$ & $\begin{array}{l}\text { Swallowing } \\
\text { rehabilitation training }\end{array}$ & $\begin{array}{l}\text { Effective rate, } \\
\text { water swallowing } \\
\text { test, Fujima Ichiro } \\
\text { dysphagia score }\end{array}$ \\
\hline Zhu (47) & 2015 & $54.1 \pm 16.6$ & $49.5 \pm 22.7$ & 30 & 30 & $\begin{array}{l}\text { Acupuncture + } \\
\text { Swallowing } \\
\text { rehabilitation training }\end{array}$ & $\begin{array}{l}\text { Swallowing } \\
\text { rehabilitation training }\end{array}$ & Effective rate \\
\hline Zhuang (48) & 2013 & $62.4 \pm 3.9$ & $61.4 \pm 4.8$ & 36 & 36 & $\begin{array}{l}\text { Acupuncture + } \\
\text { Swallowing } \\
\text { rehabilitation training }\end{array}$ & $\begin{array}{l}\text { Swallowing } \\
\text { rehabilitation training }\end{array}$ & $\begin{array}{l}\text { Fujima Ichiro } \\
\text { dysphagia score, } \\
\text { effective rate }\end{array}$ \\
\hline
\end{tabular}

SSA, standardized swallowing assessment; VFSS, video fluoroscopic swallowing study; SWAL-QOL, swallowing disorder-specific quality of life scale.

effective rate than the control group and the difference was statistically significant [relative risk $(\mathrm{RR})=1.23,95 \%$ confidence interval (CI): 1.19 to $1.27, \mathrm{P}<0.00001$; Figure 4].

\section{Drinking water test classification score}

A total of 8 studies reported the grading scores of drinking water test of patients after acupuncture treatment for dysphagia, with 475 cases in the experimental group and 454 cases in the control group. The 8 included studies were tested for heterogeneity, $\mathrm{I}^{2}=98 \%$ and $\mathrm{P}<0.00001$, indicating that there was a high degree of heterogeneity among the included studies, so a random effects model 


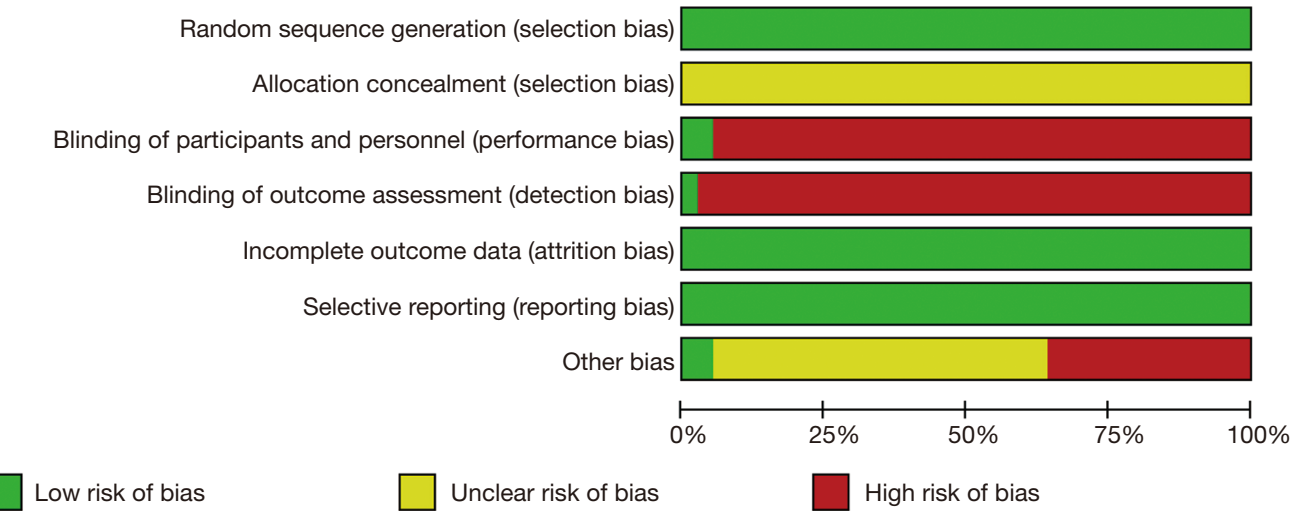

Figure 2 Bias percentage chart of included studies.

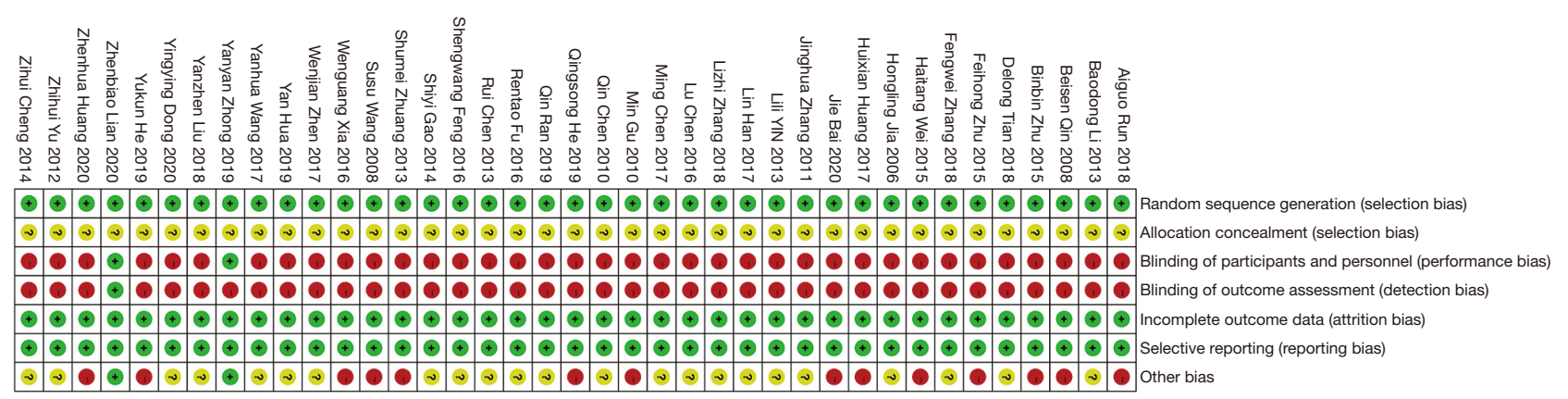

Figure 3 Bias distribution diagram of included studies. Green plus “+” means "Yes”, red plus “_” means "No”, yellow plus “?” means unclear.

was used for meta-analysis. The results revealed that the grading score of drinking water test for the experimental group was lower than the control group, and the difference was statistically significant [mean difference $(\mathrm{MD})=-0.75$, 95\% CI: -1.11 to $-0.41, \mathrm{P}<0.0001$; Figure 5]. Due to the high degree of heterogeneity among the included studies, sensitivity analysis was required. The main source of increased heterogeneity was not found through the method of elimination 1 by 1 , and the results obtained after eliminating the literature remained stable and reliable.

\section{Swallowing function score (SSA score)}

A total of 8 studies reported on the swallowing function scores of patients after acupuncture treatment for dysphagia, 346 cases in the experimental group and 334 cases in the control group. The 8 included studies were tested for heterogeneity, $\mathrm{I}^{2}=76 \%$ and $\mathrm{P}=0.0001$, indicating that there was a high degree of heterogeneity among the included studies. Therefore, a random effects model was used to conduct a meta-analysis. The results showed that the score of swallowing function of the experimental group participants was lower than that of those in the control group, and the difference was statistically significant (MD $=-4.63,95 \%$ CI: -5.68 to $-3.59, \mathrm{P}<0.00001$; Figure 6). Due to the high degree of heterogeneity among the included studies, sensitivity analysis was required. The main source of increased heterogeneity was not found through the method of elimination 1 by 1 , and the results obtained after eliminating the literature were still stable and reliable.

\section{Fujishima feeding-swallowing function rating scale} A total of 3 studies reported the Fujishima eatingswallowing rating scores of patients after acupuncture treatment for dysphagia. There were 141 cases in the experimental group and 134 cases in the control group. The 3 included studies were tested for heterogeneity, $\mathrm{I}^{2}=77 \%$ and $\mathrm{P}=0.01$, indicating that there was a high degree of heterogeneity among the included studies. Therefore, a random effects model was used for meta-analysis. The results showed that the Fujishima eating-swallowing rating score of the experimental group was higher than that of the control group, and the difference was statistically significant 


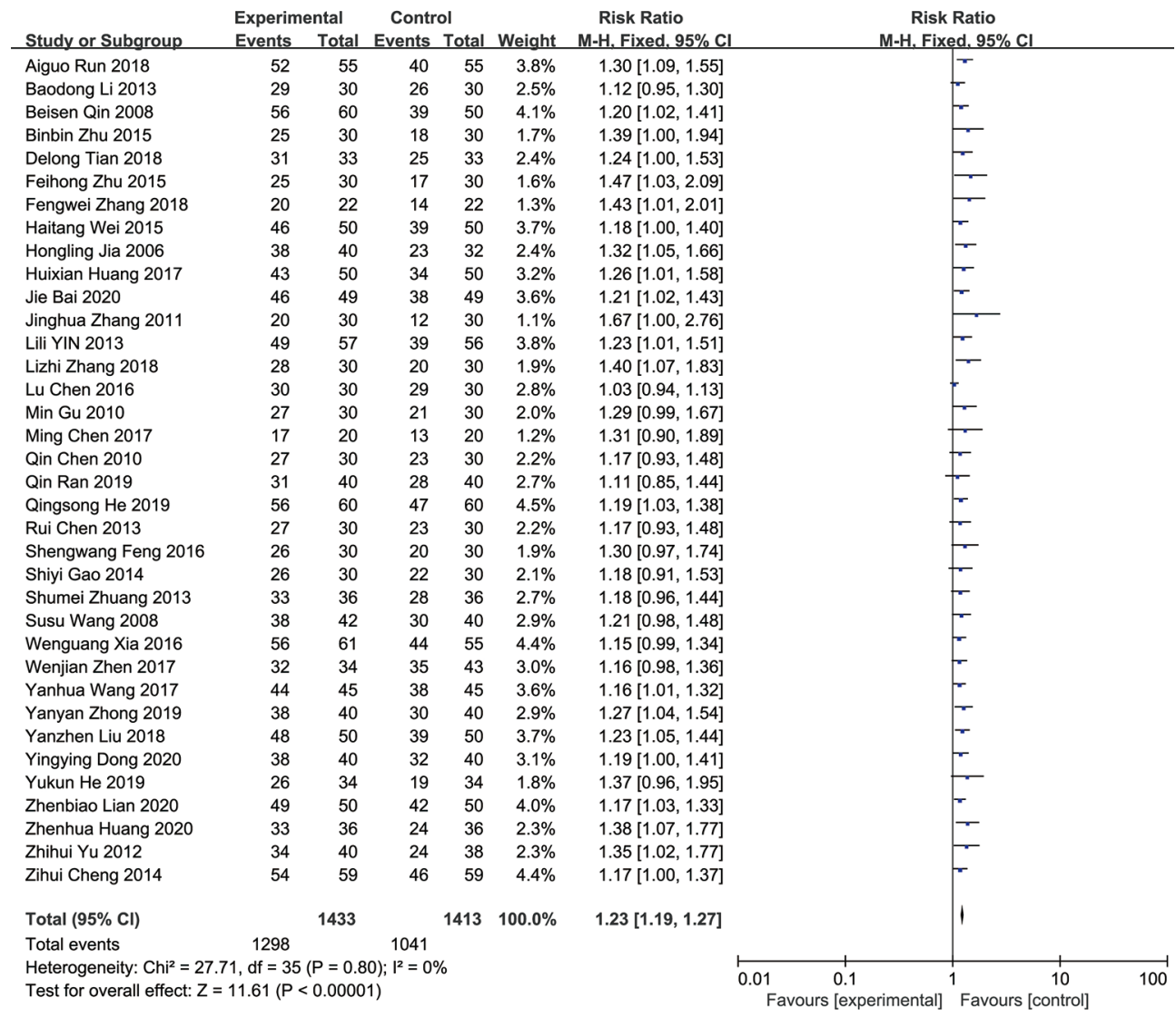

Figure 4 Forest plot of effective treatment rates for the two groups of patients. CI, confidence interval.

\begin{tabular}{|c|c|c|c|c|c|c|c|c|c|c|c|}
\hline \multirow[b]{2}{*}{ Study or Subgroup } & \multicolumn{3}{|c|}{ Experimental } & \multicolumn{3}{|c|}{ Control } & \multirow{2}{*}{\multicolumn{2}{|c|}{$\begin{array}{ll} & \text { Mean Difference } \\
\text { Weight } & \text { IV. Random. 95\% Cl }\end{array}$}} & \multirow{2}{*}{\multicolumn{3}{|c|}{$\begin{array}{l}\text { Mean Difference } \\
\text { IV. Random. } 95 \% \mathrm{Cl}\end{array}$}} \\
\hline & Mean & SD & Total & Mean & SD & Total & & & & & \\
\hline Delong Tian 2018 & 0.74 & 0.39 & 33 & 1.39 & 0.47 & 33 & $9.1 \%$ & $-0.65[-0.86,-0.44]$ & - & & \\
\hline Lu Chen 2016 & 1.33 & 0.661 & 30 & 1.83 & 0.874 & 30 & $8.4 \%$ & $-0.50[-0.89,-0.11]$ & & & \\
\hline Ming Chen 2017 & 1.35 & 0.21 & 20 & 2.31 & 0.28 & 20 & $9.3 \%$ & $-0.96[-1.11,-0.81]$ & $\pi$ & & \\
\hline Qingsong $\mathrm{He} 2019$ & 2.03 & 0.23 & 60 & 4.43 & 0.52 & 40 & $9.2 \%$ & $-2.40[-2.57,-2.23]$ & $=$ & & \\
\hline Wenjian Zhen 2017 & 2.32 & 0.24 & 44 & 3.12 & 0.16 & 43 & $9.4 \%$ & $-0.80[-0.89,-0.71]$ & $=$ & & \\
\hline Yan Hua 2019 & 1.41 & 0.63 & 49 & 1.83 & 0.74 & 49 & $8.9 \%$ & $-0.42[-0.69,-0.15]$ & - & & \\
\hline Yanyan Zhong 2019 & 1.79 & 0.72 & 40 & 2.23 & 0.74 & 40 & $8.7 \%$ & $-0.44[-0.76,-0.12]$ & & & \\
\hline Yanzhen Liu 2018 & 1.26 & 0.5 & 50 & 2.18 & 0.63 & 50 & $9.1 \%$ & $-0.92[-1.14,-0.70]$ & $=$ & & \\
\hline Yingying Dong 2020 & 1.43 & 0.42 & 40 & 1.86 & 0.54 & 40 & $9.1 \%$ & $-0.43[-0.64,-0.22]$ & & & \\
\hline Zhenbiao Lian 2020 & 1.75 & 0.19 & 50 & 2.01 & 0.33 & 50 & $9.4 \%$ & $-0.26[-0.37,-0.15]$ & $=$ & & \\
\hline Zihui Cheng 2014 & 1.55 & 0.35 & 59 & 2.02 & 0.41 & 59 & $9.3 \%$ & $-0.47[-0.61,-0.33]$ & $=$ & & \\
\hline Total $(95 \% \mathrm{Cl})$ & & & 475 & & & 454 & $100.0 \%$ & $-0.75[-1.10,-0.41]$ & & & \\
\hline $\begin{array}{l}\text { Heterogeneity: } \mathrm{Tau}^{2}= \\
\text { Test for overall effect: }\end{array}$ & $\begin{array}{l}0.33 ; \mathrm{Cl} \\
\mathrm{Z}=4.27\end{array}$ & $\begin{array}{l}\mathrm{h}^{2}=48 \\
(\mathrm{P}<0 .\end{array}$ & $\begin{array}{l}.15, \mathrm{df} \\
0001)\end{array}$ & $=10(\mathrm{P}$ & $<0.000$ & $01) ; I^{2}$ & $=98 \%$ & & -4 & 0 & \\
\hline
\end{tabular}

Figure 5 Forest diagram of the graded scores of the drinking water test after treatment in the two groups. SD, standard deviation; CI, confidence interval.

[standardized mean difference $(\mathrm{SMD})=1.92,95 \% \mathrm{CI}$ : 1.30 to $2.54, \mathrm{P}<0.00001$, Figure 7]. Due to the high degree of heterogeneity among the included studies, sensitivity analysis was required. Through the elimination method 1 by 1 , it was found that Xia Wenguang's 2016 document was the main source of increased heterogeneity, but after excluding their document, the results obtained remained stable and reliable.

\section{Dysphagia specific quality of life Scale Score}

A total of 5 studies reported on the dysphagia-specific quality of life scale scores of patients after acupuncture 


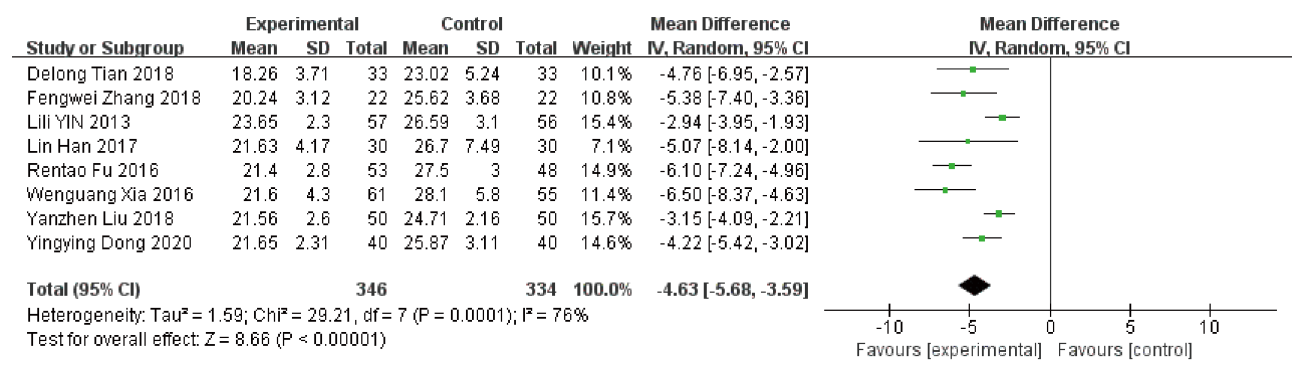

Figure 6 Forest plot of swallowing function scores of two groups of patients after treatment.

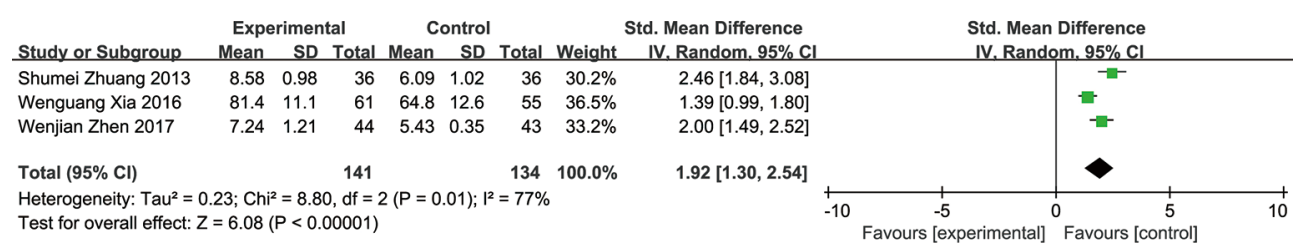

Figure 7 The forest plot of the scores of the feeding and swallowing functions of the two groups of patients after treatment.

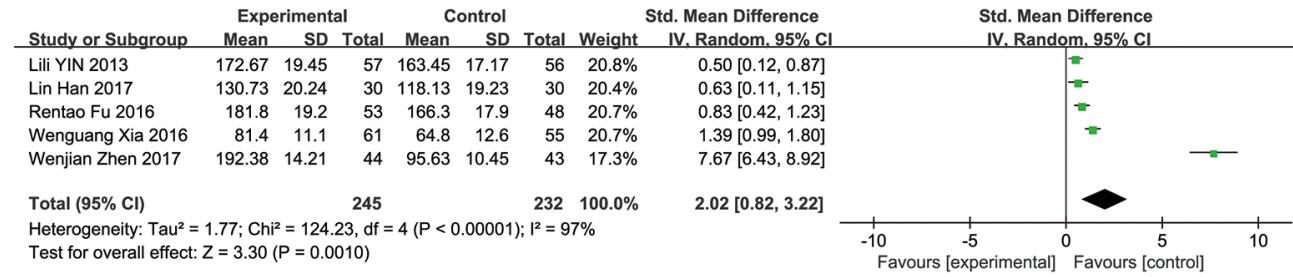

Figure 8 Forest plot of dysphagia-specific quality of life scale scores for the 2 groups of patients after treatment.

treatment for dysphagia, with 245 cases in the experimental group and 232 cases in the control group. The 5 included studies were tested for heterogeneity, $\mathrm{I}^{2}=97 \%$ and $\mathrm{P}<0.00001$, indicating that there was a high degree of heterogeneity among the included studies. Therefore, a random effects model was used to conduct a meta-analysis. The results showed that the dysphagia-specific quality of life scale score in the experimental group was higher than that of the control group, and the difference was statistically significant ( $\mathrm{SMD}=2.02,95 \% \mathrm{CI}$ : 0.82 to $3.22, \mathrm{P}=0.0001$; Figure 8). Due to the high degree of heterogeneity among the included studies, sensitivity analysis was required. The main source of increased heterogeneity was not found through the method of elimination 1 by 1 , and the results obtained after eliminating the literature were still stable and reliable.

\section{TV fluoroscopy swallowing function test}

A total of 5 studies reported on the VFSS of patients with dysphagia treated by acupuncture, with 177 cases in the experimental group and 177 cases in the control group. The 5 included studies were tested for heterogeneity, $\mathrm{I}^{2}=65 \%$ and $\mathrm{P}=0.02$, indicating that there was heterogeneity among the included studies. Therefore, a random effects model was used for meta-analysis. The results showed that the VFSS of the experimental group was higher than that in the control group, the difference was statistically significant ( $\mathrm{MD}=2.53,95 \%$ CI: 1.89 to $3.17, \mathrm{P}<0.00001$; Figure 9). Due to the high degree of heterogeneity among the included studies, sensitivity analysis was required. The main source of increased heterogeneity was not found through the method of elimination 1 by 1 , and the results obtained after eliminating the literature remained stable and reliable.

\section{Publication bias}

Evaluation of publication bias was performed for the included studies that met the indicators of 10 articles 


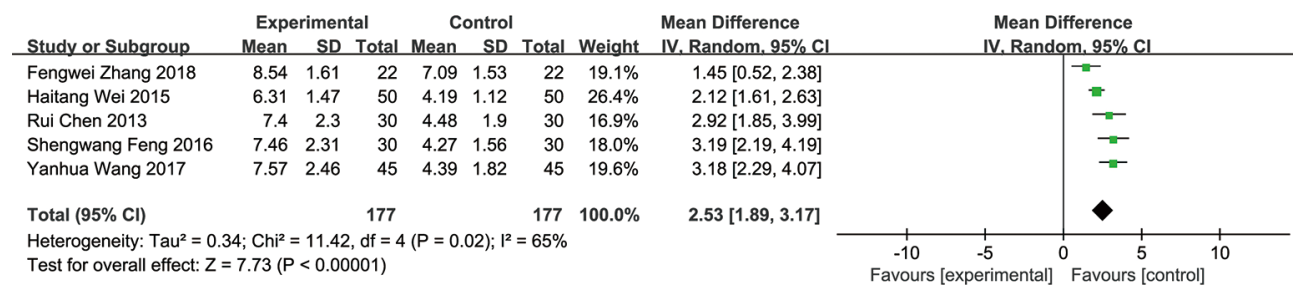

Figure 9 Forest plot of video fluoroscopic swallowing study (VFSS) after treatment in the two groups.

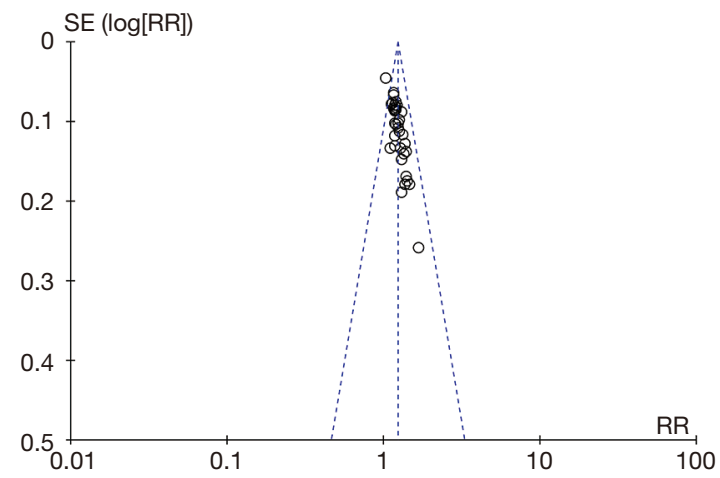

Figure 10 The funnel chart of the treatment efficiency of the two groups of patients. RR, risk ratio; SE, standard error.

(treatment effective rate). Through visual inspection of the funnel chart, it was found that the distribution of the points was more symmetrical, and the possibility of publication bias was less, indicating that the included studies were more comprehensive. See Figure 10 for details.

\section{Discussion}

After a stroke, patients may show dysphagia, which can persist for a long time. Due to long-term food restriction, patients are prone to malnutrition and lung infections. Traditional Chinese medicine respectively classifies dysphagia after stroke into categories with different characteristics. The etiology and pathogenesis of dysphagia after stroke are based on many conditions $(49,50)$. There is a close connection between the meridians of traditional Chinese medicine and the throat. If the meridians are not smooth and the "blood" is insufficient, swallowing disorders will result. Acupuncture has a long history in the treatment of dysphagia after stroke. Through the development and inheritance of generations of Chinese physicians, a wealth of treatment experience has been accumulated. Acupuncture has the functions of dredging the meridians, regulating the qi, running the blood, regulating the internal organs, clearing the throat, and rejuvenating the mind (51). Modern medicine has found that the acupuncture point locations of Fengchi (GB20), Yifeng (TH17), and Lianquan (CV23) are closely related to the nerve endings of the glossopharyngeal, vagus, facial, and hypoglossal nerves of the human body. After acupuncture is used to stimulate the nerve, the nerve impulse released by it can strengthen the corresponding neural reflexes (52). It is precisely because acupuncture therapy has the above-mentioned effects, that so many scholars use acupuncture therapy to treat swallowing disorders after stroke.

This meta-analysis included 39 randomized controlled studies, including 3078 patients. Through the integration of data in the literature, in the case of a large sample size, it was found that acupuncture combined with swallowing rehabilitation training can significantly improve the treatment efficacy of patients, which is consistent with the conclusions of most authors. Modern medicine has found that after acupuncture treatment for patients with dysphagia after stroke, the blood circulation of the vertebrobasilar artery can be significantly improved, and the latency and amplitude of brainstem auditory evoked potentials are also improved (53-55). Acupuncture therapy is effective in treating dysphagia, possibly because acupuncture reestablishes the connection between the upper motor neuron and the medullary motor nerve cell nucleus, and ultimately restores the function of the central nervous system (56). This meta-analysis also evaluated the efficacy of acupuncture in the treatment of dysphagia from multiple aspects. The results of the Kubota drinking water test showed that acupuncture can significantly reduce the time required for patients to drink $30 \mathrm{~mL}$ of water or time from drinking to coughing. The SSA scale score, Fujishima feeding-swallowing function rating scale, and SWALQOL scale score also reflected that the patient's swallowing function was significantly improved after acupuncture treatment. Therefore, this meta-analysis further confirmed 
the effectiveness of acupuncture therapy in the treatment of dysphagia after stroke by integrating large sample data.

The studies included in this meta-analysis were all RCTs, and the conclusions had high evidence strength, especially in terms of treatment efficacy. However, there were some obvious limitations in this study. In addition to more research on the effective rate of treatment, drinking water test classification, SSA score, Fujishima feeding-swallowing function grade score, and SWAL-QOL score, VFSS and other indicators included relatively few studies, included a small number of patients, and the evidence derived from these data was relatively weak. Since these indicators are scored by the corresponding standard scale, they are particularly important for the evaluation of swallowing dysfunction after stroke. Therefore, follow-up studies are required to design more comprehensively the assessment of improvement in patients' symptoms of dysphagia.

\section{Acknowledgments}

Funding: This work was supported by Guangxi Traditional Chinese Medicine Appropriate Technology Development and Promotion Project (GZXY20-16); Guangxi Traditional Chinese Medicine Science and Technology Project (GZZC16-48); Qingxiu District Scientific Research and Technology Development Project (2013S18); Project of Guangxi University of traditional Chinese Medicine (2019QN033).

\section{Footnote}

Reporting Checklist: The authors have completed the PRISMA reporting checklist. Available at http://dx.doi. org/10.21037/apm-21-499

Conflicts of Interest: All authors have completed the ICMJE uniform disclosure form (available at http://dx.doi. org/10.21037/apm-21-499). The authors have no conflicts of interest to declare.

Ethical Statement: The authors are accountable for all aspects of the work in ensuring that questions related to the accuracy or integrity of any part of the work are appropriately investigated and resolved.

Open Access Statement: This is an Open Access article distributed in accordance with the Creative Commons Attribution-NonCommercial-NoDerivs 4.0 International
License (CC BY-NC-ND 4.0), which permits the noncommercial replication and distribution of the article with the strict proviso that no changes or edits are made and the original work is properly cited (including links to both the formal publication through the relevant DOI and the license). See: https://creativecommons.org/licenses/by-nc-nd/4.0/.

\section{References}

1. Cramer SC, Chopp M. Recovery recapitulates ontogeny. Trends Neurosci 2000;23:265-71.

2. Rathore SS, Hinn AR, Cooper LS, et al. Characterization of incident stroke signs and symptoms: findings from the atherosclerosis risk in communities study. Stroke 2002;33:2718-21.

3. Joundi RA, Martino R, Saposnik G, et al. Predictors and Outcomes of Dysphagia Screening After Acute Ischemic Stroke. Stroke 2017;48:900-6.

4. Martino R, Foley N, Bhogal S, et al. Dysphagia After Stroke: Incidence, Diagnosis, and Pulmonary Complications. Stroke 2005;36:2756-63.

5. Yang HZ. Observation on the curative effect of Ditan Decoction combined with low-frequency to mediumfrequency electric stimulation in the treatment of dysphagia after stroke of wind-phlegm-stasis. Modern J Integr Trad Chin Western Med 2017;31:53-5.

6. Luo GL, Yang SW, Lu QZ, et al. Clinical Observation of External Application of Tianjiu Paste to Lianquan Point in Treating Dysphagia After Ischemic Stroke. J Guangzhou Univ Trad Chin Med 2018;3:435-9.

7. Shang Y, Ren H, Chen Q, et al. Clinical Observation on the Treatment of Dysphagia Caused by Poststroke with Renying Acupoint Injection under the Guidance of Electromyography. Hebei J TCM 2018;4:604-7.

8. Meng FZ, Wen K, Gao H, et al. Analysis of the curative effect of electromyography biofeedback combined with rehabilitation training on dysphagia after cerebral infarction. Neu Inj Funct Recons 2017;5:445-6.

9. Jia YB, Wang JM, Liu XF. Clinical Observation on Acupuncture Combined with Diagnosis and Treatment of Swallowing Miriam Speech Therapy Post-stroke Dysphagia. Liaoning J TCM 2009;10:1776-7.

10. Bai J, Liu D, Jia L, et al. Rehabilitation effect analysis of acupuncture and moxibustion combined with swallowing function training in treating dysphagia caused by stroke. Refle Rehab Med 2020,7:43-4.

11. Chen L, Zhang GM. Efficacy Study of Acupuncture and Moxibustion on Dysphagia after Stroke. JETCM 
2016;6:1111-3.

12. Chen M, Sun DJ. Clinical Observation of Swallowing Rehabilitation Staged Acupuncture Treatment on Dysphagia after Stroke. Hainan Med 2017;9:1504-5.

13. Chen Q, Xia WG, Chen BG, et al. Clinical study on acupuncture treatment of dysphagia caused by pseudobulbar palsy after stroke. Neimenggu TCM 2015;5:118-9.

14. Chen R, Xiao CQ, Xie H, et al. Observation on Therapeutic Effect of 60 Cases of Swallowing Disorder after Apoplexy Treated Mainly by Acupuncture. Inte J TCM 2013;5:450-1.

15. Cheng ZH, Zhai XF, Ji FS, et al. Acupuncture combined with rehabilitation training to treat 59 patients with dysphagia due to cerebrovascular diseases. Henan TCM 2014;3:426-7.

16. Dong YY. Study on the effect of acupuncture and swallowing training on swallowing dysfunction after stroke. Practical Integrated Chinese and Western Medicine Clinic 2020;6:59-60.

17. Feng SW, Cao SH, Du SJ, et al. Acupuncture combined with swallowing training for post-stroke dysphagia: a randomized controlled trial. Chinese Acupuncture 2016;4:347-50.

18. Fu RT. Acupuncture combined with conventional cerebrovascular disease treatment plan plus rehabilitation therapy to treat 53 cases of dysphagia after stroke. Chinese Medicine Research 2016;2:56-8.

19. Gao SY, He SS. Clinical Observation on the Treatment of 30 Cases of Swallowing Dysfunction after Stroke with Nape Three Needles and Swallowing Function Training. Hunan J TCM 2014;5:77-8.

20. Gu M, Gu XD, Yao YH, et al. Acupuncture combined with rehabilitation training to treat 30 cases of dysphagia after stroke. Chinese Medicine Technology 2010;5:461-2.

21. Han L, Gao Y. Therapeutic Observation of Acupuncture plus Rehabilitation Training for Post-stroke Dysphagia. Shanghai J Acupunc 2017;8:910-3.

22. He YK. Study on acupuncture and swallowing training for treatment of dysphagia after stroke. Medical Diet and Health 2019;16:84-8.

23. He QS, Yang LF, Wang WB. Acupuncture Treatment of Dysphagia Caused by Ischemic Stroke. J TCM 2019;7:1551-4.

24. Hua Y, Shu XM. Effect of early training combined with acupuncture on swallowing function score of patients with dysphagia after stroke. Shandong TCM 2019;5:435-8.

25. Huang HX, Yang XQ, Zhang HF, et al. An analysis of treating dysphagia after stoke by acupuncture plus swallowing training. CJCM 2017;25:27-8.

26. Huang ZH, Ruan YL, Liu B. Application of acupuncture and rehabilitation training in the treatment of dysphagia after stroke . Shenzhen J Inte TCM West Med 2020;4:20-1.

27. Jia HL, Zhang YC. Treatment of 40 postapoplectic dysphagia patients by acupuncture plus rehabilitation training. Shanghai J Acupunc 2006;7:5-6.

28. Li BD, Bai J, Gao WB, et al. The effect of neck acupuncture combined with swallowing training on swallowing dysphagia after stroke.Chin J Phys Med Rehabil 2013;1:31-2.

29. Lian ZB, Liang Q, Wang HM, et al. Curative effects of acupuncture and moxibustion and swallowing training in treatment of dysphagia after stroke. China Med Phar 2020;6:29-31.

30. Liu YZ. Analysis of curative effect of acupuncture/ moxibustion combined with rehabilitation function training in the treatment of dysphagia after stroke. PJCM 2018;2:163-6.

31. Qin PS, Liu KY, Wang D, et al. Observation on Therapeutic Effect of Acupuncture Combined with Rehabilitation Training on Swallowing Dysphagia after Stroke. Chin J Integr Med Cardio-/Cerebrovascular Dis 2008;3:274-6.

32. Ran Q, Yin SZ, Mi XJ, et al. Clinical Study on Acupuncture on Pseudobulbar Paralysis after Stroke. JETCM 2019;2:273-5.

33. Yan AG. Clinical Observation on Acupuncture and Moxibustion Combined with Traditional Chinese Medicine Rehabilitation Training in Treating Dysphagia after Stroke. Chin Med Moder Dist Edu 2018;5:122-4.

34. Tian DL, Meng FF, Chen HF, et al. Clinical effect of Xingnao acupuncture method and swallowing rehabilitation training in the treatment of dysphagia after stroke. Chin J Prim Med Pharm 2018;3:287-90.

35. Wang SS, Yang HF, Xu ZH, et al. Clinical Observation on Treatment of 42 Cases of Dysphagia after Stroke with Acupuncture on Qi Meridian. J New Chin Med 2008;5:71-2.

36. Wang YH, Yang GF, Zhou HX et al. Clinical Observation of Acupuncture plus Rehabilitation Training for Poststroke Deglutition Disorder. Shanghai J Acu-mox 2017;6:663-7.

37. Wei HT, Peng T, Yang D, et al. Clinical Study of Acupuncture Combined with Rehabilitation Training in the Treatment of Dysphagia after Stroke. JETCM 
2015;10:1808-10.

38. Xia W, Zheng C, Xia J, et al. Post-stroke dysphagia treated with acupuncture of meridian differentiation: a randomized controlled trial. Zhongguo Zhen Jiu 2016;36:673-8.

39. Yin LL. A Clinical Study on Acupuncture Combined with Rehabilitation Therapy for 57 Cases of Swallowing Disorders after Stroke. J TCM 2013;9:766-8.

40. Yu ZH, Hu JF. Observations on the Efficacy of Acupuncture plus Rehabilitation Training in Treating Post-stroke Dysphagia. Shanghai J Acu-mox 2012;9:642-4.

41. Zhang FW. Evaluation of the effect of acupuncture combined with swallowing training in treating dysphagia after stroke. J Hubei Univ Sci Tech (Med Sci) 2018;2:162-4.

42. Zhang JH, Li JY, Zhao Y, et al. Clinical Observation of Dysphgia after Stroke by Tongnao Huoluo Acupuncture Therapy with Swallowing Training. J Liaoning Univ Tradi Chin Med 2011;12:188-9.

43. Zhang LZ, Xu NG, Li RL, et al. Clinical Observation of Tong Du Tiao Shen Needling in Treating Post-stroke Deglutition Disorders. Shanghai J Acu-mox 2018:140-3.

44. Zhen WJ, Liu Y, Li N, et al. Observation on Therapeutic Effect of Acupuncture Synchronous Swallowing Rehabilitation Training on Swallowing Disorders After Cerebral Hemorrhage. JETCM 2017;10:1859-61.

45. Zhong YY, Li JH, Pan FF, et al. Clinical Study of Acupuncture Combined with Deglutition Training for Dysphagia after Stroke. J New Chin Med 2019;1:201-4.

46. Zhu BB, Zhao CY. After Swallowing Training Combined with Acupuncture to Treat Stroke Dysphagia Randomized Controlled Study. J Prac Trad Chin Internal Med 2015;11:152-3.

47. Zhu FH, Wu ZY, Zheng JF, et al. Observation on the clinical effect of three sublingual acupuncture combined

Cite this article as: Lu Y, Chen Y, Huang D, Li J. Efficacy of acupuncture for dysphagia after stroke: a systematic review and meta-analysis. Ann Palliat Med 2021;10(3):3410-3422. doi: 10.21037/apm-21-499 with swallowing function training on dysphagia after stroke. J Chin Physi 2015;17:586-7.

48. Zhuang SM, Lv HB, Liu JQ. Observation on the effect of acupuncture combined with rehabilitation training on dysphagia after stroke. Chin J Basic Med In TCM 2013;7:847-8.

49. Song SC, Lu Z, Wang ZY, et al. Research progress on acupuncture treatment of dysphagia after stroke. Chin J Integr Med Cardio-/Cerebrovascular Dis 2013;12:1511-3.

50. Zhou RX, Zhang CH. Clinical Progress of Acupuncture Treatment for Dysphagia after Stroke. JCAM 2011;11:67.

51. Liu MX, Li TC. Acupuncture treatment of 36 patients with pseudobulbar palsy after stroke. J Changchun Univ TCM 2008;3:302.

52. Deng XX, Wu C. "Xingnao Kaiqiao" Acupuncture Therapy Combined with Vitalstim Swallowing Therapeutic Apparatus in Treatment of Dysphagia of Stroke. J Chin Med 2017;32:466-9.

53. Zhang PD. Comparative observation on the effect of acupuncture and swallowing training in auxiliary treatment of dysphagia during stroke recovery. Proceedings of the first dysphagia forum 2009:827-31.

54. Chu JM, Gao WB. Item for the impact of TCD in patients with pseudobulbar palsy. JCAM 2005;8:4-6.

55. Chu JM, Gao WB. The impact of items on BAEP in patients with pseudobulbar palsy. Chin Arch TCM 2006;11:2150-1.

56. Zhang CH, Bian JL, Meng ZH, et al. Tongguan Liqiao acupuncture therapy improves dysphagia after brainstem stroke. Neural Regen Res 2016;11:285-91.

(English Language Editor: J. Jones) 